OPEN

SUBJECT AREAS:

CADHERINS

MEMBRANE PROTEINS

Received

21 July 2014

Accepted

20 October 2014

Published

6 November 2014

Correspondence and requests for materials should be addressed to M.K. (masashi.kishi@ gmail.com)

\title{
Vangl2 Regulates E-Cadherin in Epithelial Cells
}

\author{
Tadahiro Nagaoka' ${ }^{1}$ Ayumu Inutsuka ${ }^{2}$, Khadiza Begum ${ }^{3}$, Khandakar musabbir bin hafiz ${ }^{3} \&$ Masashi Kishi
}

'Laboratory of Molecular Neuroimaging, Graduate School of Medical and Dental Sciences, Niigata University, Niigata, Niigata 951-8510, Japan, ${ }^{2}$ Department of Neuroscience II, Research Institute of Environmental Medicine, Nagoya University, Furo-cho, Chikusa-ku, Nagoya 464-8601, Japan, ${ }^{3}$ Department of Microbiology and Hygiene, Bangladesh Agricultural University, Mymensingh 2202. Bangladesh.

E-cadherin belongs to the classic cadherin subfamily of calcium-dependent cell adhesion molecules and is crucial for the formation and function of epithelial adherens junctions. In this study, we demonstrate that Vangl2, a vertebrate regulator of planar cell polarity (PCP), controls E-cadherin in epithelial cells.

E-cadherin co-immunoprecipitates with Vangl2 from embryonic kidney extracts, and this association is also observed in transfected fibroblasts. Vangl 2 enhances the internalization of E-cadherin when overexpressed. Conversely, the quantitative ratio of E-cadherin exposed to the cell surface is increased in cultured renal epithelial cells derived from Vangl $2^{L t /+}$ mutant mice. Interestingly, Vangl2 is also internalized through protein traffic involving Rab5- and Dynamin-dependent endocytosis. Taken together with recent reports regarding the transport of Frizzled3, MMP14 and nephrin, these results suggest that one of the molecular functions of Vangl2 is to enhance the internalization of specific plasma membrane proteins with broad selectivity. This function may be involved in the control of intercellular PCP signalling or in the PCP-related rearrangement of cell adhesions.

$\mathrm{P}$ lanar cell polarity $(\mathrm{PCP})$ is defined as uniform orientation of a group of cells within a plane of epithelium ${ }^{1-3}$, and it occurs during tissue morphogenetic events such as alignment of cochlear hair cells $s^{4}$, epidermal hair patterning ${ }^{5}$, and convergent extension movements of lateral mesoderm ${ }^{6}$. Planar polarization is mediated through patterned cell movements and coordinated transformation of the cell shapes, and these changes depend, in part, on the extensive remodelling of cell-cell junctions ${ }^{7,8}$. Therefore, to understand the molecular mechanism underlying the establishment of PCP, it is important to reveal which cell adhesion molecules are involved and how they are regulated in a spatiotemporally precise manner.

Establishment of PCP is mediated by the Wnt/PCP pathway, a branch of noncanonical Wnt signalling, which controls gene expression changes and cytoskeletal dynamics in a transmembrane receptor Frizzled-dependent manner ${ }^{3}$. In the planar polarized epithelium, Frizzled and Dishevelled form a protein complex that confronts the other PCP complex composed of Strabismus/Vang and Prickle on the other side of the cell adhesion sites. These core PCP factors also regulate cell adhesion cooperatively with the Flamingo/Celsr atypical cadherins that mediate homophilic cell-cell adhesion ${ }^{9}$. The PCP regulators exhibit punctate localizations at the adherens junctions of Drosophila wing epithelia ${ }^{10}$. In addition, Celsr1 is enriched at the adherens junctions between the neuroepithelial cells of the neural plate to induce its polarized bending ${ }^{11}$. The PCP factors localize at adherens junctions, which are remodelled during planar polarization ${ }^{12,13}$. However, the link between adherens junction molecules and PCP factors is still obscure. For example, whether classic cadherins, which are the main cell adhesion molecules at adherens junctions, actively remodel the cell-cell junctions in response to the PCP signal or whether they are passively regulated by the Flamingo/Celsr-related rearrangement of cell adhesion is unknown.

We have recently reported that Vang-like-2 (Vangl2), a mammalian PCP regulator of Vang family proteins, associates with $\mathrm{N}$-cadherin ${ }^{14}$, a neural subtype of classic cadherins crucial for the formation of the dendritic spines and synaptic junctions in the vertebrate $\mathrm{CNS}^{15,16}$. Vangl2 enhances endocytosis of postsynaptic N-cadherin through this molecular association and is also required for the normal development of dendritic spines. Ncadherin and Vangl2 form protein complex under negative regulation by their respective primary binding partner, $\beta$-catenin and Prickle2, suggesting that Vangl2 precisely controls the cell surface levels of $\mathrm{N}$-cadherin by this conditional protein association to mediate accurate development of synaptic junctions. Although classic cadherins are structurally homologous ${ }^{17}$, certain subtype-specific properties have been reported ${ }^{18}$. In fact, whether Vangl2 is directly involved in the regulation of other classic cadherins has not been addressed. 
Endocytic regulation of E-cadherin plays an important role in the PCP-related formation, rearrangement, and maturation of cell adhesions. During the gastrulation of zebrafish embryos, intracellular trafficking of E-cadherin is controlled by the Wnt/PCP pathway stimulated by Wnt $11^{19}$. In Drosophila, development of the wing or tracheal epithelia requires polarized trafficking of E-cadherin-containing vesicles during the PCP-dependent remodelling of cell-cell junctions ${ }^{20}$. Because Vangl2 directly controls N-cadherin trafficking $^{14}$, this PCP-related endocytosis of E-cadherin may be also mediated by Vang family proteins. In the present study, we demonstrate complex formation and functional association of E-cadherin with Vangl2 in mammalian kidney epithelial cells. Vangl2mediated regulation of E-cadherin transport may play a role in epithelial morphogenesis of renal tubules, which is under the control of PCP signalling ${ }^{21,22}$.

\section{Results}

Vangl2 Forms a Protein Complex with E-Cadherin. To determine whether Vangl2 forms protein complex only with $\mathrm{N}$-cadherin or also with other classic cadherins, we analysed lysates of HEK293T cells transfected with E-cadherin and GFP-Vangl2 expression vectors using immunoprecipitation (IP). Western blot (WB) analysis indicated that GFP-Vangl2 coprecipitates with E-cadherin (Figure 1a) and vice versa (Figure 1b). The molecular association was not observed with YFP-tagged CD82, another tetraspanin highly expressed in the developing epithelia ${ }^{23}$ in the parallel

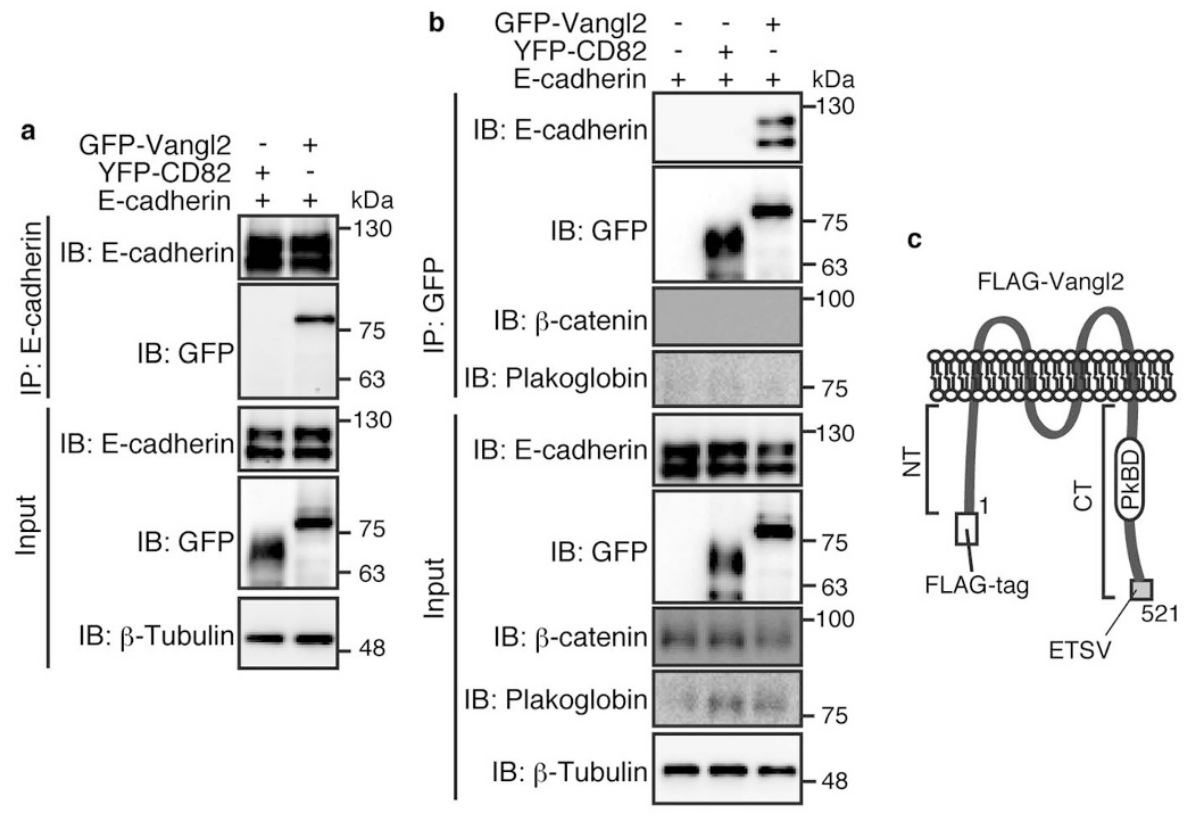

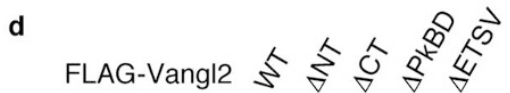

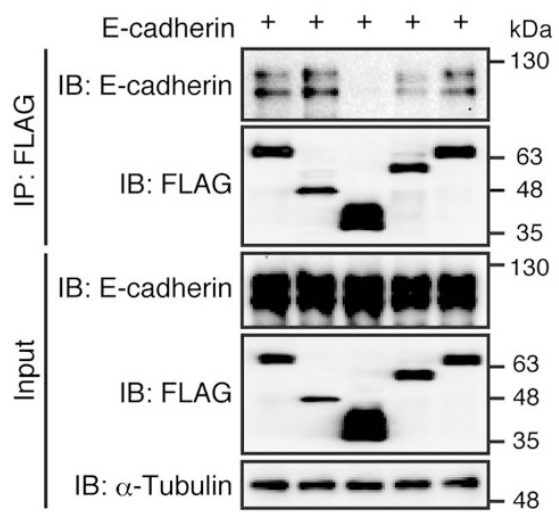

g

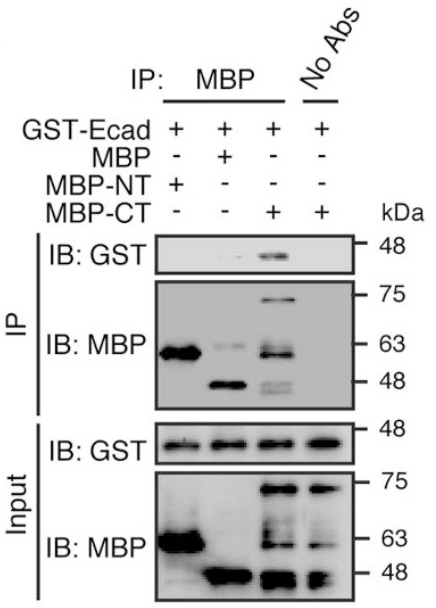

Figure 1 Association of E-cadherin with Vangl2. (a, b, d, f) HEK293T cells were transfected with the indicated expression constructs, and the cell lysates as well as IPs were analysed by WB using the indicated Abs. IP was performed using (a) $\alpha$-E-cadherin, (b) $\alpha$-GFP, or (d, f) $\alpha$-FLAG Abs. The protein band of E-cadherin with higher molecular weight indicates the immature form with propeptide. (c, e) Schematic representations of the mutated constructs of Vangl2 (c) and E-cadherin (e) used in the co-IP experiments shown in (d) and (f), respectively. (g) In vitro binding between recombinant E-cadherin and Vangl2. GST-Ecad incubated with either MBP-NT, nonfused MBP or MBP-CT, was immunoprecipitated using $\alpha$-MBP Abs and protein G sepharose. The Inputs (0.5\%) as well as IPs (approximately 30\%) were separated with SDS-PAGE and analysed by WB using the indicated Abs. Significant degradation of MBP-CT occured during the purification for some unknown reason. The right lane indicates the no Ab control. Note the specific precipitation of GST-Ecad by MBP-CT. WB images were captured using the same experimental condition for each series of experiments and for each kind of antibodies. The full-length images of the WB analyses presented in Figure 1 are included in Supplementary Figure S3. IP (immunoprecipitates), IB (immunoblot), WT (wild-type Vangl2), $\Delta$ NT (Vangl2 lacking N-terminal intracellular domain), $\Delta \mathrm{CT}$ (Vangl2 lacking the C-terminal intracellular domain), $\triangle \mathrm{PkBD}$ (Vangl2 lacking the Prickle-binding domain), $\Delta$ ETSV (Vangl2 lacking the PDZ-binding motif at the C-terminal end), S/A (E-cadherin with all serine residues within the $\beta$-catenin-binding region substituted by alanine), $\triangle \mathrm{BD}$ (E-cadherin lacking the catenin-binding region). 
experiment (Figure 1a and b). We confirmed the specificities of the antibodies (Abs) utilized in the IP experiments (see Supplementary Figure 1).

We next determined which region of the Vangl2 protein is required for the complex formation (Figure 1c and $\mathrm{d}$ ). Deletion of the N-terminal intracellular domain did not affect the coprecipitation. In contrast, the C-terminal intracellular domain (ICD) was required. Further, deletion of the Prickle-binding region ${ }^{24}$ greatly diminished the association $(28.4 \pm 1.2 \%$, compared to WT; $\mathrm{n}=3$ experiments). On the other hand, deletion of the ETSV PDZ-binding motif ${ }^{25}$ had a less significant effect $(87.1 \pm 1.5 \%$, compared to WT; $\mathrm{n}=3$ experiments).

Because association with Vangl2 requires the normal $\beta$-cateninbinding region of $\mathrm{N}$-cadherin ${ }^{26,27}$, we examined whether the corresponding region of the E-cadherin ICD is essential for the formation of the E-cadherin-Vangl2 complex (Figure 1e and f). Deletion of the $\beta$-catenin-binding region as well as alanine substitution of a stretch of serine residues within this region ${ }^{26,28}$ (Figure 1e) abolished the protein association (Figure 1f). We confirmed that the cell surface expression of the mutant forms of Vangl2 or E-cadherin was not greatly affected in comparison with that of the wild-type proteins (see Supplementary Figure 1).

These results indicate that the normal association of classic cadherins with Vangl2 requires the $\beta$-catenin-binding domain of $\mathrm{E}$ cadherin as well as the Prickle-binding domains of Vangl2. These regions may be important for the molecular interaction or structural stability of the respective proteins.

We further evaluated whether purified recombinant E-cadherin and Vangl2 interact in vitro (Figure $1 \mathrm{~g}$ ). The GST fusion protein of the E-cadherin intracellular domain (ICD) (GST-Ecad) and the MBP-fused N-terminal (MBP-NT) and C-terminal ICDs (MBP$\mathrm{CT}$ ) of Vangl2 were bacterially expressed and purified using glutathione- and amylose-conjugated resins, respectively. GST-Ecad was mixed with MBP-NT, nonfused MBP or MBP-CT and subjected to co-IP using $\alpha$-MBP Abs. GST-Ecad was precipitated only when incubated with MBP-CT. Although we cannot exclude the possibility that other factors influence this protein-protein interaction or that their transmembrane regions also play important roles in complex formation, these results suggest that the E-cadherin ICD is capable of directly binding to the Vangl2 C-terminus.

Complex Formation between E-cadherin and Vangl2 is Restricted by $\boldsymbol{\beta}$-Catenin or Prickle2. Because normal association between Ecadherin and Vangl2 requires these respective binding domains, we determined whether cotransfection of $\beta$-catenin or Prickle 2 affects the complex formation.

HEK293T cells were transfected with expression constructs of Ecadherin and FLAG-tagged Vangl2. The cell lysates were subjected to IP using $\alpha$-FLAG Abs and subsequent WB (Figure 2a and c).

The amount of E-cadherin protein that coprecipitated with Vangl2 decreased as the amount of cotransfected GFP- $\beta$-catenin expression vector increased (Figure $2 \mathrm{a}$ and b; $0.2 \mu \mathrm{g}$ : $0.49 \pm 0.03$ a.u.; $\mathrm{p}=0.004$, $0.5 \mu$ g: $0.27 \pm 0.03$ a.u.; $p=0.003,1.0 \mu$ g: $0.18 \pm 0.02$ a.u.; $p=0.001$; $\mathrm{n}=3$ experiments). Similarly, when cotransfected GFP-Prickle2 expression vector increased, a dose-dependent decrease in Vangl2associated E-cadherin was observed (Figure $2 \mathrm{c}$ and d; $0.2 \mu \mathrm{g}$ : $0.75 \pm$ 0.05 a.u.; $\mathrm{p}=0.049,0.5 \mu \mathrm{g}$ : $0.42 \pm 0.04$ a.u.; $\mathrm{p}=0.007,1.0 \mu \mathrm{g}: 0.28 \pm$ 0.05 a.u.; $\mathrm{p}=0.008 ; \mathrm{n}=3$ experiments).

Because $\beta$-catenin is expressed at a significant level in HEK293T cells, we also determined whether silencing of endogenous $\beta$-catenin affects the formation of the E-cadherin-Vangl2 complex (see Supplementary Figure 2). Co-introduction of the $\beta$-cateninshRNA construct ${ }^{14}$ significantly increased the protein level of E-cadherin associated with Vangl2 (2.17 \pm 0.22 a.u.; $\mathrm{p}=0.03 ; \mathrm{n}=3$ experiments). These results indicate that the association between E-cadherin and Vangl2 is suppressed by $\beta$-catenin or Prickle2. In other words, E-cadherin and Vangl2 are capable of forming protein complexes that are sensitive to the expression levels of their respective primary binding partners.

Vangl2 Enhances the Internalization of E-Cadherin. In the previous study, we demonstrated that internalization of $\mathrm{N}$ cadherin is enhanced by Vangl2 in transfected cells as well as in cultured hippocampal neurons ${ }^{14}$. In the present study, we determined the effects of Vangl2 on cell surface expression of Ecadherin. a

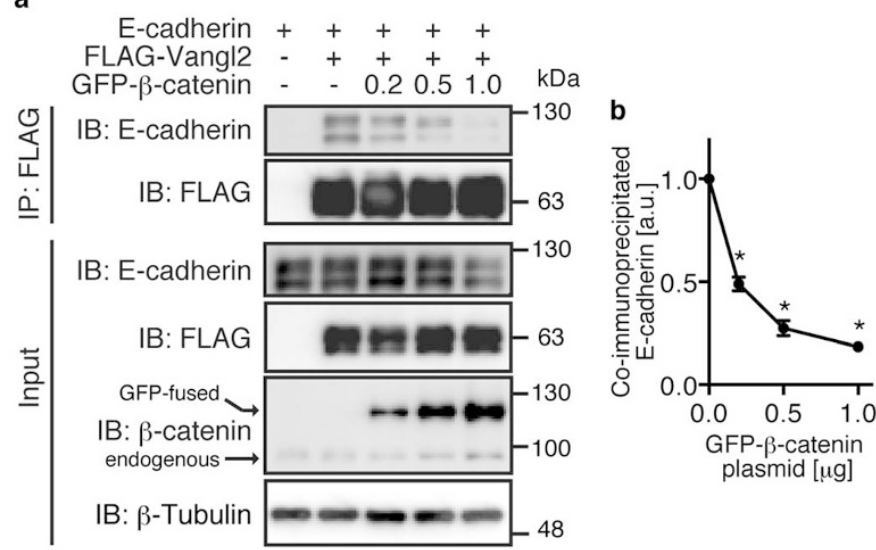

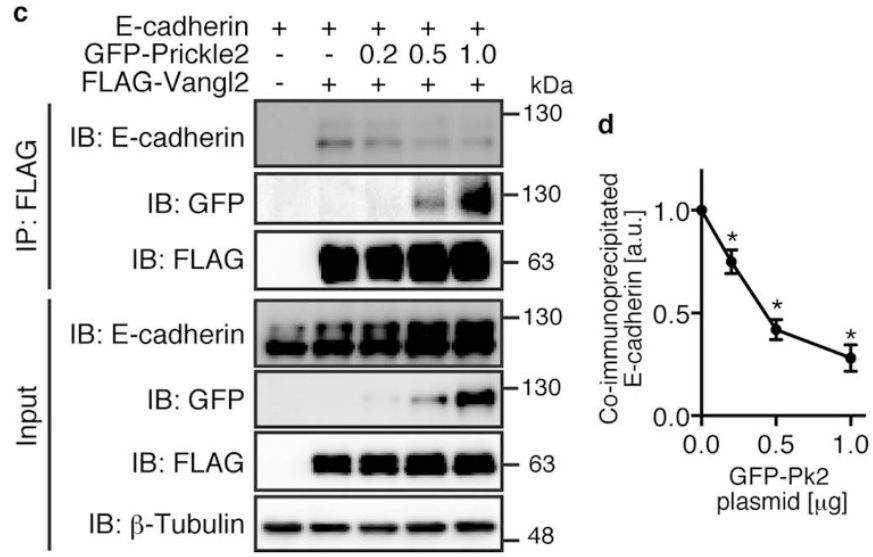

Figure 2 Inhibition of complex formation between E-cadherin and Vangl2 by either $\boldsymbol{\beta}$-catenin or Prickle2. (a, c) HEK293T cells were transfected with the indicated expression plasmids, and the cell lysates as well as IPs were analysed by WB. IP was performed using $\alpha$-FLAG Abs. Total DNA amount was adjusted to $3.0 \mu \mathrm{g}$ for each well using control GFP expression vector. (b, d) Line graphs showing the ratio of the E-cadherin levels included in the IPs to those in the total cell lysates. The ratios were calculated according to the immunosignals quantified using ImageJ. The line graphs (b) and (d) were prepared according to the WB analyses (a) and (c), respectively. For quantification of E-cadherin levels, only signal strength of the protein band corresponding to the mature form (lower molecular weight) was used. Note the dose-dependent reduction of the precipitated levels of E-cadherin by increased DNA amount of $\beta$-catenin $(a, b)$ or Prickle2 $(c, d)$. WB images were captured using the same experimental condition for each series of experiments and for each kind of antibodies. The full-length images of the WB analyses presented in Figure 2 are included in Supplementary Figure S4. Data are presented as mean \pm SEM. Significant differences $(\mathrm{p}<0.05)$ versus a one stage earlier group calculated using Student's t test are marked with *. a.u.: arbitrary unit. 
Cell surface proteins expressed on HEK293T cells transfected with the indicated expression vectors (Figure $3 \mathrm{a}$ ) were biotinylated and precipitated using avidin-coated beads ${ }^{29}$. The protein samples included in the precipitates as well as in the total cell lysates were analysed by WB. Treatment of the cells with 4 mM EGTA, which chelates the extracellular calcium ions, induced a reduction in cell surface levels of E-cadherin ${ }^{30}$. Similarly, cotransfection of Vangl2 decreased the E-cadherin levels exposed to the cell surface (Figure $3 \mathrm{a}$ and $\mathrm{b}$ ). In these experiments, the EGTA treatment, but not Vangl2 transfection, induced the internalization of transferrin receptors (TfR; Figure 3a and c), indicating the functional specificity of Vangl2.
The enhanced internalization of E-cadherin may be due to increased endocytosis, decreased exocytosis or toxic effects on protein transport. To address this issue, we further introduced dominant-negative forms of Rab5 $5^{31}$ (RFP-DN-Rab5) or Dynamin ${ }^{32}$ (DN-Dynamin1-GFP). Cotransfection of these constructs of endocytosis inhibitors clearly rescued the cell surface expression of E-cadherin (Figure $3 a$ and $b$ ), demonstrating that enhanced internalization of E-cadherin by Vangl2 involves endocytic transport. In this experiment, Vangl2 was also internalized through a pathway involving Rab5 and Dynamin (Figures 3a and d). Reflecting the sensitivity to dnRab5, immunofluorescence (IF) microscopy revealed the vesicular co-localization of Rab5 with a portion of vesicles that a

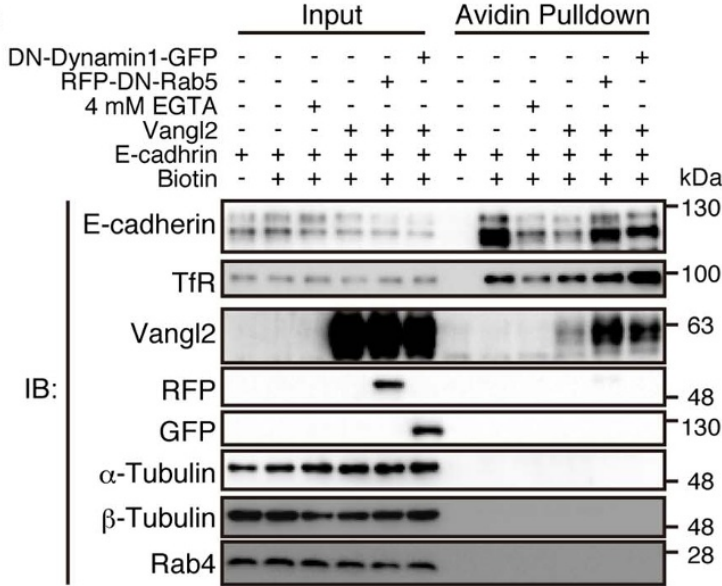

b

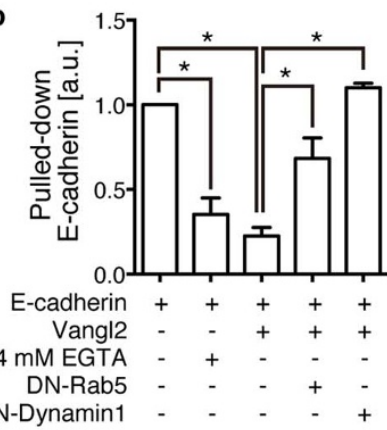

c

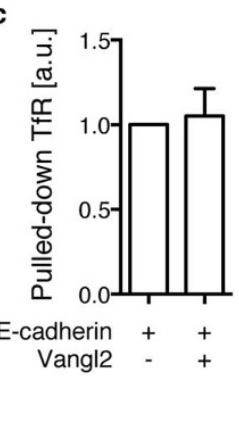

d

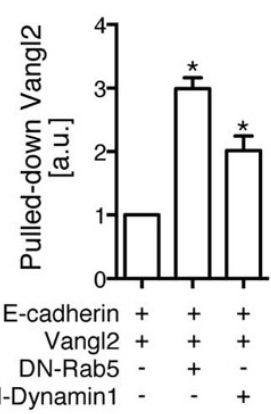

f

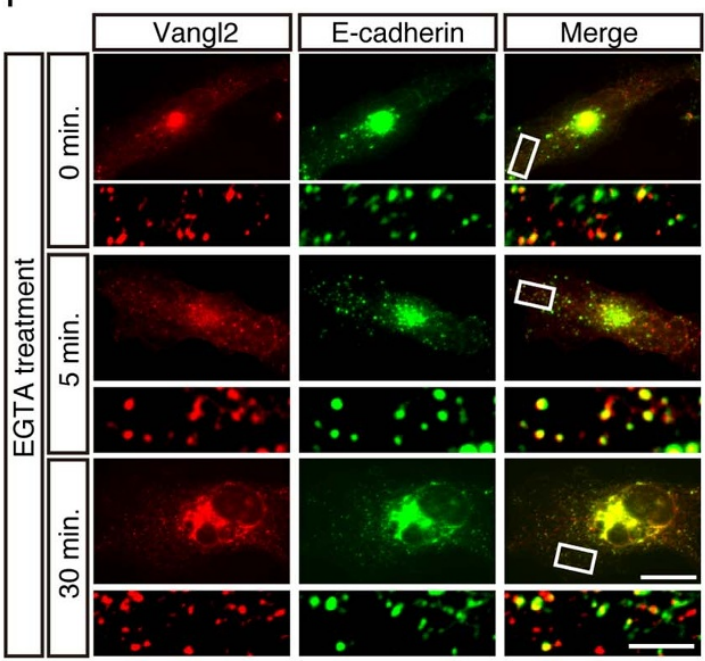

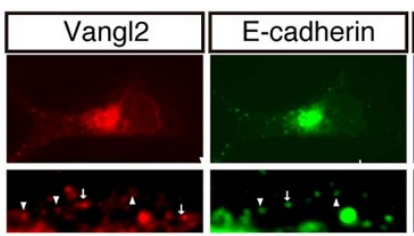

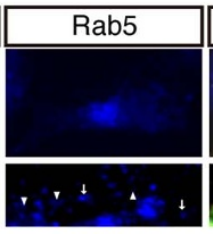

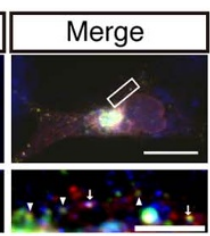

g

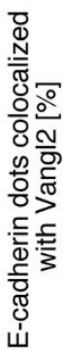

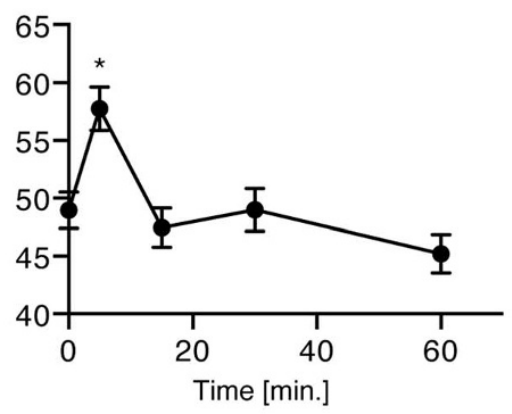

Figure 3 Regulation of cell surface levels of E-cadherin by Vangl2. (a) WB analysis of the indicated proteins included in the total cell lysates or exposed to the cell surface. The indicated expression constructs were transfected into HEK293T cells, and the cell lysates were analysed. Biotinylated surface proteins were pulled down via avidin affinity. (b-d) Quantification of the surface levels of E-cadherin (b), TfR (c) and Vangl2 (d) treated with the indicated reagents and expression constructs. The bar graphs show the ratio of the protein levels precipitated using the avidin beads to those included in the total cell lysates. (e) Co-localization of the E-cadherin (green)-Vangl2 (red) vesicles with Rab5 (blue). Expression constructs of Vangl2 and E-cadherin were transfected into HEK293T cells. E-cadherin/Vangl2 double-positive and E-cadherin/Vangl2/Rab5 triple-positive dots are indicated by arrowheads and arrows, respectively. (f) IF analysis of colocalization of transfected E-cadherin with Vangl2. The presented images were captured without EGTA treatment $(0 \mathrm{~min}$.), or 5 and 30 minutes after the EGTA treatment. (g) Line graph showing the transition of the ratio of the E-cadherin vesicles colocalized with Vangl2 IF. The images captured without EGTA treatment ( $0 \mathrm{~min}$.), or 5, 15, 30, and 60 minutes after the EGTA treatment were analysed. WB images were captured using the same experimental condition for each series of experiments and for each kind of antibodies. The full-length images of the WB analyses presented in Figure 3 are included in Supplementary Figure S5. Higher magnification of the delimited region is shown in the respective lower panels. Data are presented as mean + SEM $(b, c, d)$ or \pm SEM $(g)$. Significant differences $(p<0.05)$ versus control groups calculated using Student's t test are marked with *. a.u.: arbitrary unit. Scale bars: $20 \mu \mathrm{m}$ and $5 \mu \mathrm{m}$ for upper and lower panels, respectively. 
were positive for both E-cadherin and Vangl2 (Figure 3e; $13.3 \%, \mathrm{n}=$ 400 E-cadherin/Vangl2 double-positive dots). Because Rab5 is an early regulator of endocytosis, and because Vangl2-mediated enhancement of E-cadherin internalization is highly sensitive to dnRab5 (Figures 3a and b), the relatively rare coincidence of Rab5 IF with E-cadherin/Vangl2 double-positive dots may indicate that their co-localization is transient.

Vangl2 transfection as well as EGTA treatment enhanced E-cadherin endocytosis. We therefore analysed whether colocalization of Vangl2 with E-cadherin is affected by the EGTA treatment (Figure $3 \mathrm{f}$ and $g$ ). We counted the E-cadherin puncta associated with Vangl2 IF. Without EGTA treatment, we found that $49.0 \pm 1.6 \%(\mathrm{n}=40$ cells $)$ of the E-cadherin puncta are associated with Vangl2. Five minutes after the treatment, we observed a transient increase in the colocalization $(57.8 \pm 1.9 \% ; \mathrm{p}=0.001 ; \mathrm{n}=40$ cells $)$, which then returned to the steady state $(15 \mathrm{~min}: 47.5 \pm 1.7 \% ; \mathrm{p}=0.523,30 \mathrm{~min}: 49.0 \pm$ $1.8 \% ; \mathrm{p}=0.984,60 \mathrm{~min}: 45.2 \pm 1.7 \% ; \mathrm{p}=0.107)$. This transition in the ratio of their co-localization suggests that Vangl2 is, to a large extent, involved in the early phase of E-cadherin trafficking.

Vangl2 Associates with E-Cadherin In Vivo. To assess the significance of these transfection experiments, we examined the embryonic kidneys where the branching morphogenesis of epithelia is dependent of Vangl2 $2^{33}$. Embryonic day 19 rat kidney extracts were prepared as described in the "Methods", and subjected to IP using either $\alpha$-Vangl2 Abs or normal goat $\operatorname{IgG}^{14}$. The immunoprecipitates (IPs) were analysed by WB using the indicated Abs (Figure 4a). E-cadherin was specifically detected in the IPs of $\alpha$-Vangl2, suggesting that a small portion of Ecadherin associates with Vangl2 in vivo. In this experiment, as expected from the IP data in HEK293T cells (Figures 2a and b), $\beta$-catenin was not detected in the $\alpha$-Vangl2 IPs even when the blots were overexposed. Although Plakoglobin can substitute for $\beta$-catenin ${ }^{34}$, it was not included in the IPs. These results suggest that E-cadherin is dissociated from the catenins when it is associated with Vangl2.

Using IF microscopy, we analysed the localizations of these proteins on the horizontal section of a renal tubule from the kidney of a postnatal day $0(\mathrm{P} 0)$ mouse (Figure $4 \mathrm{~b})$. E-cadherin and Vangl2 were highly accumulated at the lateral borders between the epithelial cells (Figures $4 \mathrm{~b}$ and $\mathrm{c}$ ). When the intensities of the IF of the enlarged images were plotted along the border (Figure $4 \mathrm{c}$; white line), the signal peaks of both fluorophores were correlated to some extent. On this plot, the signal peaks of phalloidin, which primarily stain the apico-lateral region of the epithelial cells, were less significantly associated with each IF signal (Figure 4d; blue). When the IF intensities were plotted transversely to the apico-basal axis of the cell (Figure 4c; yellow line), we found that Vangl2 was distributed more intracellularly with punctate signals (Figure 4e; red). Taken together, these results indicate that, at least, the subpopulations of E-cadherin and Vangl2 co-localize in the epithelia of embryonic kidneys, primarily at cell-cell junctions.

Cell Surface Expression of E-Cadherin Is Abnormal in Epithelial Cells Derived from Vangl2 ${ }^{L p t /+}$ Mutant Kidneys. Homozygous Loop-tail (Lpt) mutation in the mouse Vangl2 gene results in embryonic lethality due to incomplete closure of the neural tube ${ }^{35}$. To determine the role of Vangl2 in the regulation of the cell surface expression of E-cadherin, we analysed cultured epithelial cells derived from the neonatal kidneys of $V a n g l 2^{L p t /+}$ or wild-type littermate mice. Because Vangl2 with the Lpt mutation is highly unstable $^{36,37}$, the total protein level of Vangl2 was significantly decreased in the mutant cultures (Figure 5a and c; $0.46 \pm 0.05$ a.u.; $\mathrm{p}=0.002 ; \mathrm{n}=4$ experiments). It is also possible that the function of the wild-type Vangl2 protein is affected by the residual $L p t$ mutant form of Vangl2 through its dominant-negative action ${ }^{38}$.

Using the same method employed for HEK293T cells (Figure 3a), we assessed the cell surface expression of E-cadherin in these primary a

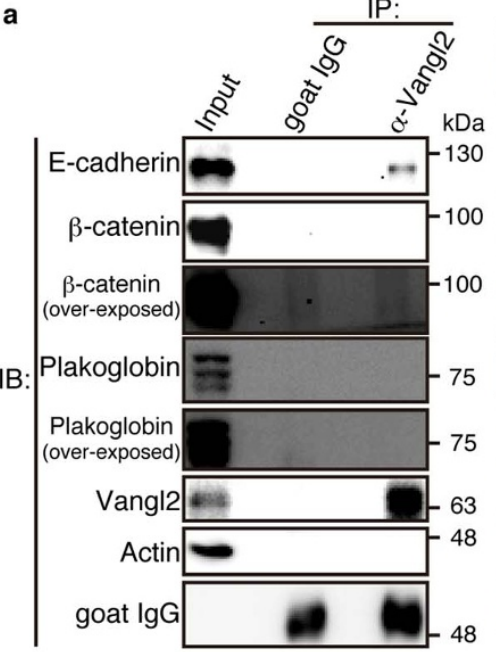

b

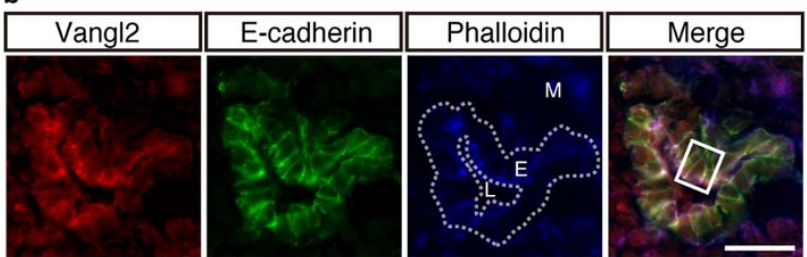

C
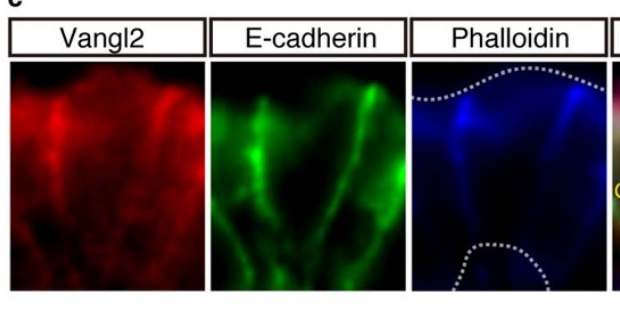

d

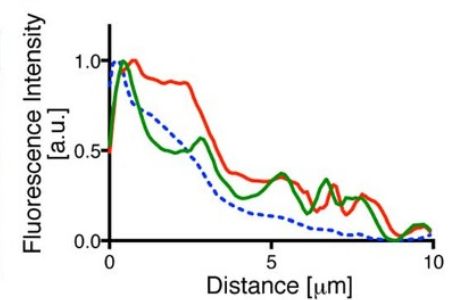

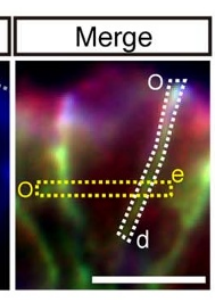

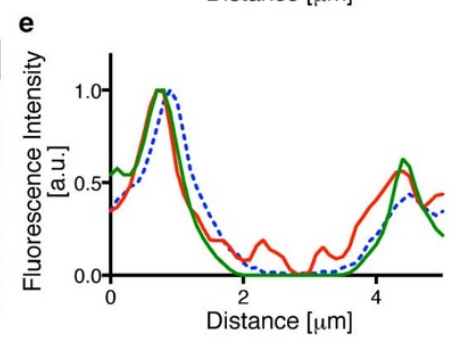

Figure $4 \mid$ E-cadherin-Vangl2 association in vivo. (a) E19 rat kidney extracts were subjected to IP using normal goat IgG or $\alpha$-Vangl2 Abs, and then to WB analysis. Note the specific precipitation of E-cadherin by $\alpha$-Vangl2. $\beta$-catenin or Plakoglobin were not detected in the IPs even when the blots were overexposed. (b, c) IF localization of Vangl2 (red) and E-cadherin (green) shown together with phalloidin staining (blue) on a cryosection of a P0 mouse kidney. E-cadherin is robustly expressed in the epithelial cells of the urinary tubule, which is horizontally cut on this section. Higher magnification of the delimited region (b) is shown in (c). Apical and basal limits of the epithelia are shown using white broken lines in the images of phalloidin staining (blue) and are displayed at the upper and lower side of the images of (c), respectively. The white characters in the phalloidin image of (b) denote the following: $M$, mesenchyme; E, epithelia; and L, lumen. (d, e) Plot profile analysis of the IF intensity along the delimited regions (c). The origin of the plot is shown as " 0 ". The signal intensities are plotted along one of the lateral borders between the epithelial cells (d; delimited with white dots) or transverse to these borders (e; delimited with yellow dots). The $\mathrm{X}$-axis indicates the distance from the origin of the plot. The $\mathrm{Y}$-axis delineates fluorescent signal intensity. For each fluorophore and each plot, the most intense signal peak was set as one arbitrary unit of the Y-axis. WB images were captured using the same experimental condition for each series of experiments and for each kind of antibodies. The full-length images of the WB analyses presented in Figure 4 are included in Supplementary Figure S6. Scale bars: $20 \mu \mathrm{m}$ and $5 \mu \mathrm{m}$ for (b) and (c), respectively. 
a

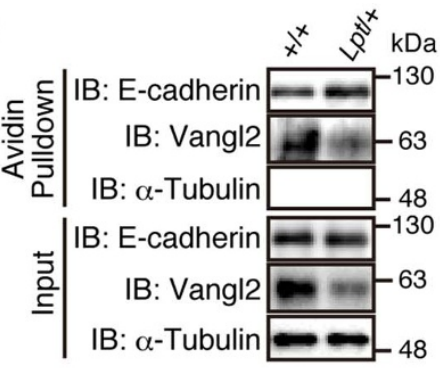

C

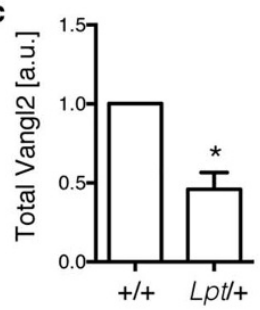

d

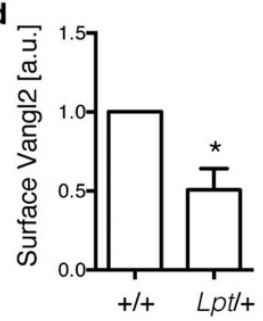

b
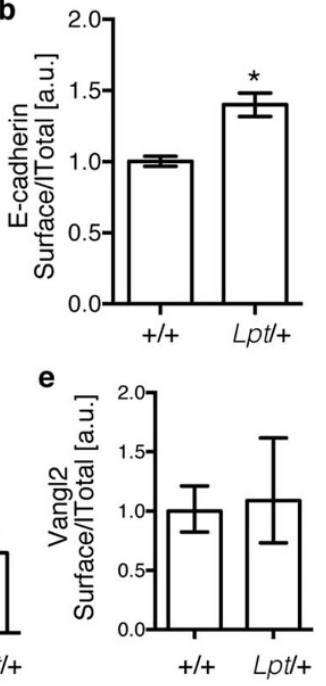
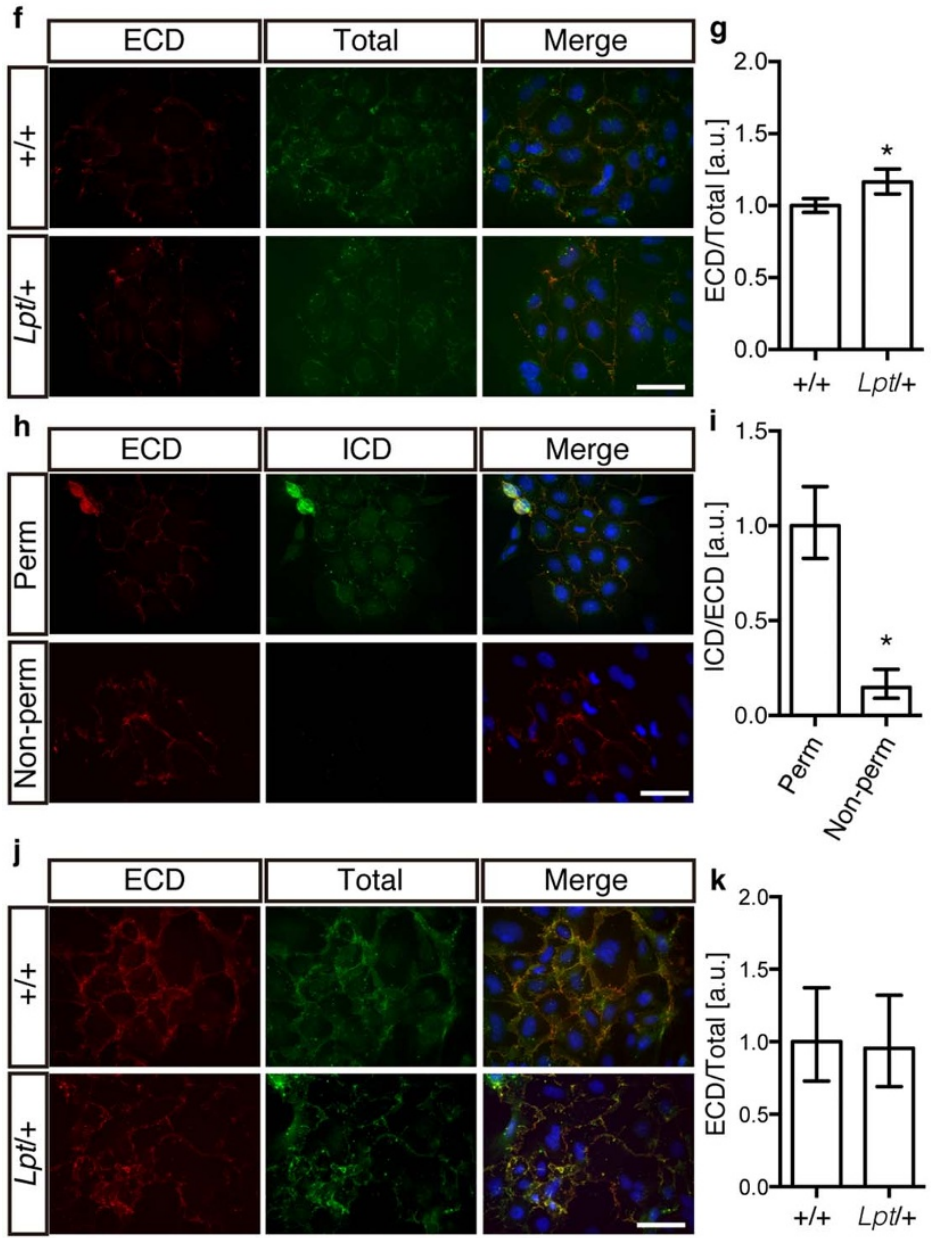

Figure 5 Increased cell surface expression of E-cadherin on the epithelial cells derived from the Vangl2 ${ }^{L p t+}$ mutant kidneys. (a) WB analysis of both biotinylated cell surface proteins and proteins included in the total cell lysates of the cultured kidney cells derived from the wild-type or Vangl2 $2^{L t /+}$ mutant mice. (b) Bar graphs showing the cell surface ratios of E-cadherin levels, which were quantified according to the immunosignal intensities shown in (a). (c-e) Quantitative analysis of Vangl2 levels in the kidney cells derived from wild-type or Vangl2 ${ }^{L t /+}$ mutant mice. The WB signal levels of total Vangl2 (c), those exposed to the cell surface (d) and their ratios (e) were compared between these two genotypes. (f, h, j) Image analysis of cultured kidney epithelial cells derived from wild-type and Vangl2 $2^{L t / /}$ mutant mice using the $\alpha$-ECD and $\alpha$-ICD Abs. $\alpha$-ECD Abs were applied before (f) or after (j) membrane permeabilization. In (h), wild-type cells were stained either with or without permeabilization for both Abs. The images were captured using the same exposure time and gain for each fluorophore and for each series of experiments. $(\mathrm{g}, \mathrm{i}, \mathrm{k})$ Bar graphs showing the relative average ratios of the signal intensities of $\alpha$-ECD and $\alpha$-ICD. The geometric mean of ratio of the wild-type (g, $\mathrm{k}$ ) and that of the permeabilized culture was set as one arbitrary unit for each experimental condition $(f),(j)$ and $(h)$. WB images were captured using the same experimental condition for each series of experiments and for each kind of antibodies. The full-length images of the WB analyses presented in Figure 5 are included in Supplementary Figure S7. Data are presented as geometric mean $\pm 95 \%$ CI (b, e, g, i, k) or mean + SEM (c, d). Significant differences (p $<0.05)$ versus control groups calculated using Mann-Whitney's U test (b, e, g, i, k) or Student's t test (c, d) are marked with *. a.u.: arbitrary unit. Scale bars: $50 \mu \mathrm{m}$. 
cultures. Consistent with the data obtained by the transfection experiments (Figures $3 a$ and $b$ ), the surface fraction of the cell lysate derived from the mutant cells, which was purified using biotin-avidin affinity, contained a significantly increased level of E-cadherin (Figures 5a and b; 139\%, 95\% CI: 118-164\%; $\mathrm{p}=0.0079 ; \mathrm{n}=5$ experiments). In this experiment, the cell surface level of Vangl2 was also decreased in the mutant cells (Figure 5d; $0.51 \pm 0.07$ a.u.; $\mathrm{p}=0.005 ; \mathrm{n}=4$ experiments), and the quantitative ratio of Vangl2 expressed on the cell surface was not significantly different between these two genotypes (Figure 5e; 109\%, 95\% CI: 73.3-162\%; p = 0.829; $\mathrm{n}=4$ experiments).

The cell surface levels of E-cadherin were also assessed by IF intensity ${ }^{39}$. The primary cultures were fixed using $4 \%$ paraformaldehyde (PFA) and stained with a rat DECMA-1 monoclonal Ab, which was raised against the extracellular domain (ECD) of E-cadherin ${ }^{40}$. Cells were then washed extensively, permeabilized with Triton X100 , and then incubated with mouse $\alpha$-E-cadherin intracellular domain (ICD) Abs. These primary Abs were detected using Alexa594 -conjugated $\alpha$-rat and Alexa-488-conjugated $\alpha$-mouse IgG secondary Abs, respectively. By this procedure, while $\alpha$-ECD Abs almost solely recognize cadherin molecules exposed to the cell surface, $\alpha$-ICD Abs detected both the cell surface and intracellular populations ${ }^{14,39}$. We calculated the ratio of total signal intensity of Alexa-594 to that of Alexa- 488 for each image pair as the quantitative ratio of Ecadherin molecules exposed to the cell surface. We then calculated the geometric means of the ratios for both genotypes, and compared them. By this image analysis, we found that the cell surface ratio of Ecadherin on the Vangl $^{L p t /+}$ epithelial cultures significantly increased (Figure 5f and g; 117\%, 95\% CI: 108-126\%; $\mathrm{p}=0.008$; $\mathrm{n}=5$ experiments), as shown for the cell surface levels of $\mathrm{N}$-cadherin on the cultured neurons ${ }^{14}$.

In the control experiments, $\alpha$-ICD Abs did not detect the ICD epitope at a comparative level without permeabilization (Figure $5 \mathrm{~h}$ and i; $14.8 \%$, 95\% CI: 9.1-24.3\%; $\mathrm{p}<0.0001$ ), indicating that membrane permeabilization is required for sufficient penetration of the Abs. Further, in the experiments in which both $\alpha$-ECD and $\alpha$-ICD were applied after permeabilization, the average ratio of both IF signals was not greatly different between the two genotypes (Figure 5j and k; 95.5\%, 95\% CI: 69.1-132\%; $\mathrm{p}=0.802 ; \mathrm{n}=5$ experiments), indicating that the permeabilization significantly enhances the penetration of not only $\alpha$-ICD but also $\alpha$-ECD Abs in these experiments.

Taken together, these results indicate that Vangl2 is required for maintaining the normal cell surface levels of E-cadherin in the kidney epithelial cells.

\section{Discussion}

The PCP factors localize to and regulate adherens junctions ${ }^{11,20}$. A possible mechanism of this regulation was shown by a genetic study of Vang12 ${ }^{41}$. Transgenic overexpression as well as genetic ablation of Vangl2 in mice resulted in aberrant formation of adherens junctions between embryonic neuroepithelial cells. In addition, MDCK cells transfected with a Vangl2 expression vector displayed decreased cellcell adhesion in hanging drop cultures, suggesting that precise regulation of Vangl2 levels is required for the appropriate formation of adherens junctions. Although Rac1-mediated cytoskeletal rearrangement is suggested to play an important role in this regulation, whether cell adhesion molecules are directly affected by Vangl2 has not been addressed. In the present study, we have demonstrated that Vangl2 associates with E-cadherin to enhance its internalization in the developing epithelial cells. The bacterially expressed E-cadherin ICD directly bind C-terminal ICD of Vangl2. It is therefore possible that this control is one of the causes of the impaired adherens junction formation in these transgenic animals ${ }^{41}$. The cell surface levels of E-cadherin on the Vangl $2^{L t / /}$-derived renal epithelial cells were indeed significantly increased in our analysis. Similarly, in
Drosophila, the PCP pathway controls recycling and turnover of Ecadherin during junctional remodelling, and E-cadherin levels increase at junctions between tracheal epithelial cells of the Strabismus/Vang mutant ${ }^{20,42}$. Although the detailed molecular mechanism is unknown, similar protein interactions shown in our mammalian studies might be involved in the Drosophila phenotype of the E-cadherin regulation.

Vangl2 is also known to induce endocytosis of several other membrane proteins. At the growing axonal tips, Vangl2 antagonizes Dishevelled1-mediated phosphorylation of Frizzled3 and enhances its endocytosis ${ }^{43}$. Through this regulation of Wnt signalling, Vangl2 mediates the precise extension of filopodia in response to chemoattractants. During the gastrulation of zebrafish embryos, the PCP pathway regulates endocytosis of the metalloproteinase MMP14 to control remodelling of the extracellular matrix ${ }^{44}$. Loss of Vangl 2 in trilobite mutants leads to increased cell surface amounts of MMP14, abnormal remodelling of extracellular matrix, and disturbance of convergent extension movements ${ }^{45}$. Further, a cell adhesion molecule expressed in the kidney podocyte, nephrin, is regulated by Vangl $2^{46}$. Activation of the PCP pathway promotes nephrin endocytosis in a clathrin- and $\beta$-arrestin-dependent manner. Depletion of Vangl2 increases the cell surface amount of nephrin and disturbs the maturation of glomeruli. How is the endocytosis of these structurally unrelated molecules enhanced by Vangl2? We deduce that Vangl2 regulates their internalization with selectivity, as the cell surface expression of TfR was not significantly affected by Vangl2 in our study (Figure $3 \mathrm{a}$ and $\mathrm{c}$ ). It should be noted that TfR is a single-pass type II membrane protein with a cytosplasmic N-terminal domain, and classic cadherins, nephrin, MMP14 and Frizzled are all singlepass type I membrane proteins with intracellular C-terminal domains. This difference in membrane topology may affect the sensitivity of transmembrane proteins to Vangl2-mediated internalization. With regard to the molecular interactions organized by Vangl2, it is suggested that MAGI2, a podocyte scaffold protein, forms a link between nephrin and Vangl2, the interaction of which may be involved in the endocytosis of nephrin ${ }^{47}$. By contrast, classic cadherins can directly bind Vangl2 dependently on their $\beta$-cateninand Prickle-binding sites ${ }^{14}$. Taken together, these results suggest that the molecular mechanism of the Vangl2-promoted endocytosis is context-dependent. Interestingly, the assembly of extracellular matrix during zebrafish gastrulation, which is controlled by the cell surface levels of MMP14, seems to be influenced by the cadherinmediated cell adhesion ${ }^{48}$. Given that the E-cadherin ICD can directly associate with Vangl2, we propose the hypothesis that regulation of the cell surface expression of classic cadherin by Vangl2 plays a primary role in multiple aspects of PCP-related remodelling of cell adhesion.

From the viewpoint of molecular evolution, a predominant role for the cadherin superfamily in the establishment of the PCP signalling pathway should be noted. There are three classes of regulators of PCP signalling: the upstream activators, the core factors, and the downstream effectors ${ }^{49}$. Other than Wnt proteins, Fat $(\mathrm{Ft})$ and Dachsous (Ds) protocadherins are known to function as the upstream activators of PCP signalling. Whereas Ft is expressed uniformly, Ds is expressed in a gradient in the Drosophila eye and wing. Because Ds acts as the ligand for Ft, Ft is activated in a gradient, providing the long-range cue for PCP activation in these tissues to establish the polarized distributions of the core PCP factors within each cell ${ }^{50}$. In mammals, Fat1, Fat4, and Dachsous1 control tubular elongation of renal epithelia through PCP signalling, preventing the development of polycystic kidney diseases ${ }^{51}$. Interestingly, both $\mathrm{Ft}$ and Ds harbour homologous regions to the intracellular $\beta$-cateninbinding domain of classic cadherins ${ }^{52}$, with which Vangl2 may physically interacts. Therefore, whether Vangl2 also associates Ft and/or Ds to form feedback loops in the PCP pathway is an issue to be addressed. As for the core PCP regulators, Fmi/Celsr proteins are 
serpentine receptor-like atypical cadherins ${ }^{9}$. Further, we have shown that classic cadherin is one of the effectors of Vangl2 ${ }^{14}$. We therefore conclude that cadherin superfamily proteins are included in all three classes of PCP regulators. This strong correlation suggests a crucial role for calcium-dependent cell adhesion in the appearance of tissue polarity during evolution. We propose that advancement of cell-cell interaction in complexity or elaboration by gene evolution of cadherin motif-containing molecules is a plausible mechanism for creating multicellular organisms with PCP-like tissue organization.

In conclusion, we observed physical and functional associations of Vangl2 with E-cadherin, which are essentially similar to that with $\mathrm{N}$-cadherin. Vangl2 positively regulates endocytosis of both $\mathrm{N}$ - and E-cadherin in a Rab5-dependent manner. Microscopically, the vesicular colocalization of cadherins with Vangl2 is approximately $50 \%$ in common ${ }^{14}$ ( $53.1 \%$ for N-cadherin; $49.0 \%$ for E-cadherin). On the other hand, we noticed a significant difference in the ratio of Rab5colocalization. While $71 \%$ of the Vangl2-colocalized N-cadherin puncta are associated with Rab5 $\mathrm{IF}^{14}$, only $13 \%$ of those of E-cadherin are Rab5-positive (Figure 3c). The high ratio of Rab5 association with the $\mathrm{N}$-cadherin-Vangl2 complex may explain why $\mathrm{N}$-cadherin undergoes a high rate of endocytosis at the postsynapse, where Vangl2 is enriched. N-cadherin, but not E-cadherin, is known to interact with the fibroblast growth factor receptor through the fourth cadherin domain $(\mathrm{EC} 4)^{53}$. The associated fibroblast growth factor receptor signalling may enable Vangl2 to recruit Rab5 to N-cadherin more efficiently than to E-cadherin. Future comparative analysis of the constituents of the cadherin-Vangl2 protein complexes would reveal the subtype-specific features of the cadherin transport during PCP-related tissue morphogenesis.

\section{Methods}

Animals. Loop-tail mutants of the $L P T / L e$ stock $^{54}$ were purchased from the Jackson Laboratory (Bar Harbor, ME), and backcrossed to C57BL/6J strain for at least 8 generations. The Animal Use and Care Committee of Niigata University authorized all animal experiments, which were performed in accordance with the NIH Guidelines for Care and Use of Laboratory Animals.

Plasmids and Antibodies. Expression constructs of Vangl2, FLAG-Vangl2, dominant-negative Rab5, GFP- $\beta$-catenin, and GFP-Prickle2 are as described ${ }^{14}$. The supplemental Excel file contains detailed information on other plasmid constructs (sheet \#1) and Abs (sheet \#2) used in this study. The plasmid constructs were sequenced to confirm the absence of sequence errors.

Tissue Culture. HEK293T cells (ATCC, Manassas, VA) were maintained in standard conditions using DMEM containing GlutaMAX (Life Technologies, Carlsbad, CA) supplemented with $10 \%$ foetal bovine serum (BioWest, Kansas City, MO) at $37^{\circ} \mathrm{C}$ in the presence of $5 \% \mathrm{CO}_{2}$ in a water-jacketed incubator. Transfections of the plasmid DNA were performed using Lipofectamine 2000 (Life Technologies) or polyethylenimine (Polysciences Inc., Warrington, PA: \#23966) according to the manufacturer's instructions. $24 \mathrm{~h}$ after transfection, cells were either treated or untreated with the indicated reagents, and then either harvested for biochemical analysis or fixed for microscopic analysis.

Primary Culture of the Mouse Kidney Cells. Both kidneys were dissected out from each P0 Vangl $2^{L p t /+}$ mutant mouse or their control littermate of wild-type, minced using razor blades, and incubated with $200 \mu \mathrm{l}$ of $0.25 \%$ trypsin in HBSS for $15 \mathrm{~min}$ at $37^{\circ} \mathrm{C}$. Loosened tissues were washed with HBSS and then passed through the P-200 pipet tips. Dissociated cells were seeded onto either coverslips coated with poly-Llysine for IF or 6-well plates for the biotinylation assay. Cells were cultured for 4 days before analysis.

Immunofluorescence Microscopy and Image Analysis. Immunostaining and image analysis of cultured cells were performed essentially as described ${ }^{14}$. Cells were fixed with $4 \%$ paraformaldehyde in phosphate-buffered saline (PBS). 20 image pairs were analysed for measurement of total IF intensity in each experiment unless otherwise stated.

Immunoprecipitation and Western Blot Analysis. IP and WB analysis were performed essentially as described ${ }^{14}$. Cell lysis buffer (1\% NP-40, $150 \mathrm{mM} \mathrm{NaCl}$, $50 \mathrm{mM}$ Tris-Cl pH 7.5, $2 \mathrm{mM}$ EDTA and 10\% glycerol, supplemented with $20 \mu \mathrm{g} / \mathrm{ml}$ leupeptin) was used for all steps of cell lysis and bead washing. For preparation of kidney tissue extracts, $\sim 24$ kidneys dissected out from E19 SD rats (Japan SLC, Hamamatsu, Shizuoka) were lysed in $2.4 \mathrm{ml}$ of cell lysis buffer by sequential passages through P-1000 pipet tips, 21-gauge needles, and 25-gauge needles. The lysates were centrifuged at $14,000 \times g$ for $10 \mathrm{~min}$ at $4^{\circ} \mathrm{C}$, and the supernatants were used as the kidney extracts. To prepare recombinant proteins of the E-cadherin ICD and Vangl2 ICDs, cDNA encoding these protein regions were PCR-amplified and ligated into pGEX-4T-1 and pMAL-c2X vectors in frame, respectively. Bacterial expression and purification of the fusion proteins were performed according to the manufacturer's instructions. For IP of the purified proteins, approximately $1.5 \mu \mathrm{g}$ of each recombinant was added to $300 \mu \mathrm{l}$ of cell lysis buffer, incubated overnight at $4{ }^{\circ} \mathrm{C}$ with rotation and treated with $\alpha$-MBP Abs and $10 \mu \mathrm{l}$ of a slurry of protein G Mag Sepharose Xtra (GE Healthcare, Waukesha, WI) overnight at $4^{\circ} \mathrm{C}$.

Cell Surface Biotinylation Assay. Cells, seeded at $8 \times 10^{5}$ per well of 6-well plates and grown for 4 days (primary kidney) or for $24 \mathrm{hr}$ after transfection (HEK293T), were

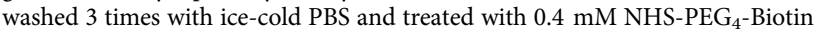
(Thermo, Waltham, MA) for $30 \mathrm{~min}$ on ice. The biotinylation reaction was quenched and excess reagents were washed out using PBS containing 0.1 M glycine. Cells were then lysed in $700 \mu \mathrm{l}$ of cell lysis buffer. Three hundred $\mu \mathrm{l}$ of the cell lysate were incubated with $5 \mu \mathrm{l}$ of High Capacity NeutrAvidin agarose resin (Thermo) for $2 \mathrm{~h}$ at $4^{\circ} \mathrm{C}$ with rotation. The resins were washed 3 times with cell lysis buffer and boiled in $2 \times$ Laemmli SDS sample buffer (Bio-Rad, Hercules, CA) supplemented with $0.1 \mathrm{M}$ DTT.

Immunofluorescent Quantification of E-Cadherin Exposed on the Cell Surface. Image analysis of the cell surface expression of cadherin was performed essentially as described ${ }^{14}$. After culturing for 4 days, the primary kidney cells were fixed with $4 \%$ PFA in PBS at $37^{\circ} \mathrm{C}$ for $15 \mathrm{~min}$, incubated with the blocking solution for $1 \mathrm{~h}$ and incubated at $4{ }^{\circ} \mathrm{C}$ for $1 \mathrm{~h}$ with the rat monoclonal Abs raised against $\alpha$-E-cadherin ECD (DECMA-1). The unbound Abs were removed by washing five times with PBS for $10 \mathrm{~min}$ each, and the plasma membranes were permeabilized using $0.1 \%$ Triton $\mathrm{X}-100$ for $10 \mathrm{~min}$. The cells were blocked again and incubated at $4^{\circ} \mathrm{C}$ for $1 \mathrm{~h}$ with mouse Abs against E-cadherin ICD (clone\#36). The $\alpha$-ECD and $\alpha$-ICD Abs bound to the primary cultures were detected using secondary Abs conjugated to different fluorophores (Alexa-594 for $\alpha$-rat IgG and Alexa-488 for $\alpha$-mouse IgG). The cellsurface and total populations of E-cadherin were identified by red and green fluorescence, respectively. All images were captured when more than 10 E-cadherinexpressing cells were clustered in the field of view using an $\times 40$ objective lens. For each series of experiments and for each fluorophore, images were captured using the same exposure time and gain without saturation using MetaMorph (Molecular Devices, Sunnyvale, CA), and the signal strength of each image was quantitatively analysed using Image (NIH, Bethesda, MD).

Immunofluorescence Analysis of Mouse Kidney. Mouse kidneys were dissected out from P0 C57BL/6J mice and freshly frozen in OCT compound (Sakura Finetek, Tokyo, Japan) using dry ice. The kidneys were sectioned at 5 - $\mu \mathrm{m}$ thickness along the dorsal-ventral axis using a cryostat (CM1850, Leica Biosystems, Nussloch, Germany) and dried on MAS-coated glass slides (Matsunami Glass, Osaka, Japan). The sectioned tissues were fixed using 4\% PFA in PBS, blocked using 10\% FBS in PBS, and sequentially incubated with the indicated antibodies accompanied by intervening washing procedures. IF images were captured and analysed as described ${ }^{14}$. To profile the signal intensity of IF along lines, Plot Profile analysis was conducted using ImageJ. The signal intensity of each plot $(0.1 \mu \mathrm{m} / \mathrm{plot})$ was statistically analysed.

Statistical Analysis. All counts and measurements were conducted in blind fashion. Each experiment was repeated at least twice and produced consistent results. The measured values from three independent experiments were used for each statistical analysis unless otherwise stated. The average ratio of one of the control samples was set as one arbitrary unit to represent the relative amounts, and used as the standard for the statistical analysis. Data are presented as mean + SEM or geometric mean $\pm 95 \%$ CI unless otherwise stated.

1. Simons, M. \& Mlodzik, M. Planar cell polarity signaling: from fly development to human disease. Annu Rev Genet 42, 517-540, doi:10.1146/ annurev.genet.42.110807.091432 (2008)

2. Vladar, E. K., Antic, D. \& Axelrod, J. D. Planar cell polarity signaling: the developing cell's compass. Cold Spring Harb Perspect Biol 1, a002964, doi:10.1101/ cshperspect.a002964 (2009).

3. Wallingford, J. B. Planar cell polarity and the developmental control of cell behavior in vertebrate embryos. Annu Rev Cell Dev Biol 28, 627-653, doi:10.1146/ annurev-cellbio-092910-154208 (2012).

4. Montcouquiol, M. et al. Identification of Vangl2 and Scrb1 as planar polarity genes in mammals. Nature 423, 173-177, doi:10.1038/nature01618 (2003).

5. Devenport, D. \& Fuchs, E. Planar polarization in embryonic epidermis orchestrates global asymmetric morphogenesis of hair follicles. Nat Cell Biol 10, 1257-1268, doi:10.1038/ncb1784 (2008).

6. Wallingford, J. B. et al. Dishevelled controls cell polarity during Xenopus gastrulation. Nature 405, 81-85, doi:10.1038/35011077 (2000).

7. Bertet, C., Sulak, L. \& Lecuit, T. Myosin-dependent junction remodelling controls planar cell intercalation and axis elongation. Nature 429, 667-671, doi:10.1038/ nature02590 (2004)

8. Walck-Shannon, E. \& Hardin, J. Cell intercalation from top to bottom. Nat Rev Mol Cell Biol 15, 34-48, doi:10.1038/nrm3723 (2014). 
9. Usui, T. et al. Flamingo, a seven-pass transmembrane cadherin, regulates planar cell polarity under the control of Frizzled. Cell 98, 585-595 (1999).

10. Strutt, H., Warrington, S. J. \& Strutt, D. Dynamics of core planar polarity protein turnover and stable assembly into discrete membrane subdomains. Dev Cell 20, 511-525, doi:10.1016/j.devcel.2011.03.018 (2011).

11. Nishimura, T., Honda, H. \& Takeichi, M. Planar cell polarity links axes of spatial dynamics in neural-tube closure. Cell 149, 1084-1097, doi:10.1016/ j.cell.2012.04.021 (2012).

12. Rauzi, M., Lenne, P. F. \& Lecuit, T. Planar polarized actomyosin contractile flows control epithelial junction remodelling. Nature 468, 1110-1114, doi:10.1038/ nature09566 (2010).

13. Zallen, J. A. \& Blankenship, J. T. Multicellular dynamics during epithelial elongation. Semin Cell Dev Biol 19, 263-270, doi:10.1016/j.semcdb.2008.01.005 (2008).

14. Nagaoka, T. et al. The Wnt/Planar Cell Polarity Pathway Component Vangl2 Induces Synapse Formation through Direct Control of N-Cadherin. Cell Rep 6 , 916-927, doi:10.1016/j.celrep.2014.01.044 (2014).

15. Togashi, H. et al. Cadherin regulates dendritic spine morphogenesis. Neuron 35 , 77-89 (2002).

16. Uchida, N., Honjo, Y., Johnson, K. R., Wheelock, M. J. \& Takeichi, M. The catenin/ cadherin adhesion system is localized in synaptic junctions bordering transmitter release zones. J Cell Biol 135, 767-779 (1996).

17. Yagi, T. \& Takeichi, M. Cadherin superfamily genes: functions, genomic organization, and neurologic diversity. Genes Dev 14, 1169-1180 (2000)

18. Suyama, K., Shapiro, I., Guttman, M. \& Hazan, R. B. A signaling pathway leading to metastasis is controlled by N-cadherin and the FGF receptor. Cancer Cell 2, 301-314 (2002).

19. Ulrich, F. et al. Wnt11 functions in gastrulation by controlling cell cohesion through Rab5c and E-cadherin. Dev Cell 9, 555-564, doi:10.1016/ j.devcel.2005.08.011 (2005)

20. Warrington, S. J., Strutt, H. \& Strutt, D. The Frizzled-dependent planar polarity pathway locally promotes E-cadherin turnover via recruitment of RhoGEF2. Development 140, 1045-1054, doi:10.1242/dev.088724 (2013).

21. Karner, C. M. et al. Wnt9b signaling regulates planar cell polarity and kidney tubule morphogenesis. Nat Genet 41, 793-799, doi:10.1038/ng.400 (2009).

22. Lienkamp, S. S. et al. Vertebrate kidney tubules elongate using a planar cell polarity-dependent, rosette-based mechanism of convergent extension. Nat Genet 44, 1382-1387, doi:10.1038/ng.2452 (2012).

23. Odintsova, E., Sugiura, T. \& Berditchevski, F. Attenuation of EGF receptor signaling by a metastasis suppressor, the tetraspanin CD82/KAI-1. Curr Biol 10, 1009-1012 (2000).

24. Jenny, A., Darken, R. S., Wilson, P. A. \& Mlodzik, M. Prickle and Strabismus form a functional complex to generate a correct axis during planar cell polarity signaling. EMBO J 22, 4409-4420, doi:10.1093/emboj/cdg424 (2003).

25. Wolff, T. \& Rubin, G. M. Strabismus, a novel gene that regulates tissue polarity and cell fate decisions in Drosophila. Development 125, 1149-1159 (1998).

26. Ozawa, M., Baribault, H. \& Kemler, R. The cytoplasmic domain of the cell adhesion molecule uvomorulin associates with three independent proteins structurally related in different species. EMBO J 8, 1711-1717 (1989).

27. Stappert, J. \& Kemler, R. A short core region of E-cadherin is essential for catenin binding and is highly phosphorylated. Cell Adhes Commun 2, 319-327 (1994)

28. McEwen, A. E., Maher, M. T., Mo, R. \& Gottardi, C. J. E-cadherin phosphorylation occurs during its biosynthesis to promote its cell surface stability and adhesion. Mol Biol Cell 25, 2365-2374, doi:10.1091/mbc.E14-01-0690 (2014).

29. Suzuki, M. et al. Defective membrane expression of the $\mathrm{Na}(+)-\mathrm{HCO}(3)(-)$ cotransporter NBCe1 is associated with familial migraine. Proc Natl Acad Sci U S A 107, 15963-15968, doi:10.1073/pnas.1008705107 (2010).

30. Le, T. L., Yap, A. S. \& Stow, J. L. Recycling of E-cadherin: a potential mechanism for regulating cadherin dynamics. J Cell Biol 146, 219-232 (1999).

31. Bohdanowicz, M., Balkin, D. M., De Camilli, P. \& Grinstein, S. Recruitment of OCRL and Inpp5B to phagosomes by Rab5 and APPL1 depletes phosphoinositides and attenuates Akt signaling. Mol Biol Cell 23, 176-187, doi:10.1091/mbc.E11-06-0489 (2012).

32. Lee, E. \& De Camilli, P. Dynamin at actin tails. Proc Natl Acad Sci U S A 99 , 161-166, doi:10.1073/pnas.012607799 (2002).

33. Yates, L. L. et al. The planar cell polarity gene Vangl 2 is required for mammalian kidney-branching morphogenesis and glomerular maturation. Hum Mol Genet 19, 4663-4676, doi:10.1093/hmg/ddq397 (2010).

34. Fukunaga, Y. et al. Defining the roles of beta-catenin and plakoglobin in cell-cell adhesion: isolation of beta-catenin/plakoglobin-deficient F9 cells. Cell Struct Funct 30, 25-34 (2005).

35. Kibar, Z. et al. Ltap, a mammalian homolog of Drosophila Strabismus/Van Gogh, is altered in the mouse neural tube mutant Loop-tail. Nat Genet 28, 251-255, doi:10.1038/90081 (2001)

36. Gravel, M., Iliescu, A., Horth, C., Apuzzo, S. \& Gros, P. Molecular and cellular mechanisms underlying neural tube defects in the loop-tail mutant mouse. Biochemistry 49, 3445-3455, doi:10.1021/bi902180m (2010).
37. Guyot, M. C. et al. A novel hypomorphic Looptail allele at the planar cell polarity Vangl2 gene. Dev Dyn : an official publication of the American Association of Anatomists 240, 839-849, doi:10.1002/dvdy.22577 (2011).

38. Yin, H., Copley, C. O., Goodrich, L. V. \& Deans, M. R. Comparison of phenotypes between different vangl 2 mutants demonstrates dominant effects of the Looptail mutation during hair cell development. PloS One 7, e31988, doi:10.1371/ journal.pone.0031988 (2012).

39. Willingham, M. C. Fluorescence labeling of surface antigens of attached or suspended tissue-culture cells. Methods Mol Biol 588, 143-151, doi:10.1007/9781-59745-324-0_17 (2010).

40. Vestweber, D. \& Kemler, R. Identification of a putative cell adhesion domain of uvomorulin. EMBO J 4, 3393-3398 (1985).

41. Lindqvist, M. et al. Vang-like protein 2 and Racl interact to regulate adherens junctions. J Cell Sci 123, 472-483, doi:10.1242/jcs.048074 (2010).

42. Classen, A. K., Anderson, K. I., Marois, E. \& Eaton, S. Hexagonal packing of Drosophila wing epithelial cells by the planar cell polarity pathway. Dev Cell 9 , 805-817, doi:10.1016/j.devcel.2005.10.016 (2005).

43. Shafer, B., Onishi, K., Lo, C., Colakoglu, G. \& Zou, Y. Vangl2 promotes Wnt/ planar cell polarity-like signaling by antagonizing Dvl1-mediated feedback inhibition in growth cone guidance. Dev Cell 20, 177-191, doi:10.1016/ j.devcel.2011.01.002 (2011).

44. Williams, B. B. et al. VANGL2 regulates membrane trafficking of MMP14 to control cell polarity and migration. J Cell Sci 125, 2141-2147, doi:10.1242/ jcs.097964 (2012)

45. Jessen, J. R. et al. Zebrafish trilobite identifies new roles for Strabismus in gastrulation and neuronal movements. Nat Cell Biol 4, 610-615, doi:10.1038/ ncb828 (2002).

46. Babayeva, S. et al. Planar cell polarity pathway regulates nephrin endocytosis in developing podocytes. J Biol Chem 288, 24035-24048, doi:10.1074/ jbc.M113.452904 (2013).

47. Babayeva, S., Zilber, Y. \& Torban, E. Planar cell polarity pathway regulates actin rearrangement, cell shape, motility, and nephrin distribution in podocytes. Am J Physiol Renal Physiol 300, F549-560, doi:10.1152/ajprenal.00566.2009 (2011)

48. Dohn, M. R., Mundell, N. A., Sawyer, L. M., Dunlap, J. A. \& Jessen, J. R. Planar cell polarity proteins differentially regulate extracellular matrix organization and assembly during zebrafish gastrulation. Dev Biol 383, 39-51, doi:10.1016/ j.ydbio.2013.08.027 (2013)

49. Benzing, T., Simons, M. \& Walz, G. Wnt signaling in polycystic kidney disease J Am Soc Nephrol: JASN 18, 1389-1398, doi:10.1681/ASN.2006121355 (2007).

50. Matis, M. \& Axelrod, J. D. Regulation of PCP by the Fat signaling pathway. Genes Dev 27, 2207-2220, doi:10.1101/gad.228098.113 (2013).

51. McNeill, H. Planar cell polarity and the kidney. J Am Soc Nephrol : JASN 20 2104-2111, doi:10.1681/ASN.2008111173 (2009).

52. Clark, H. F. et al. Dachsous encodes a member of the cadherin superfamily that controls imaginal disc morphogenesis in Drosophila. Genes Dev 9, 1530-1542 (1995).

53. Kim, J. B. et al. N-Cadherin extracellular repeat 4 mediates epithelial to mesenchymal transition and increased motility. J Cell Biol 151, 1193-1206 (2000)

54. Smith, L. J. \& Stein, K. F. Axial elongation in the mouse and its retardation in homozygous looptail mice. J Embryol Exp Morphol 10, 73-87 (1962).

\section{Acknowledgments}

The authors are grateful to Motomi Tamura for assistance and to Dr. Michihiro Igarashi for support for laboratory equipment.

\section{Author contributions}

T.N. performed all experiments. A.I., K.B. and K.M. contributed to molecular biological experiments. M.K. and T.N. conceived the experiments and wrote the paper.

\section{Additional information}

Supplementary information accompanies this paper at http://www.nature.com/ scientificreports

Competing financial interests: The authors declare no competing financial interests. How to cite this article: Nagaoka, T., Inutsuka, A., Begum, K., hafiz, K.m.b. \& Kishi, M. Vangl2 Regulates E-Cadherin in Epithelial Cells. Sci. Rep. 4, 6940; DOI:10.1038/srep06940 (2014).

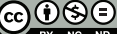

This work is licensed under a Creative Commons Attribution-NonCommerciaNoDerivs 4.0 International License. The images or other third party material in this article are included in the article's Creative Commons license, unless indicated otherwise in the credit line; if the material is not included under the Creative Commons license, users will need to obtain permission from the license holder in order to reproduce the material. To view a copy of this license, visit http:// creativecommons.org/licenses/by-nc-nd/4.0/ 